\title{
Topical thermal therapy with hot packs suppresses physical inactivity-induced mechanical hyperalgesia and up-regulation of NGF
}

\author{
Tatsuki Nakagawa $^{1,2} \cdot$ Shin-ichiro Hiraga ${ }^{1,2} \cdot$ Kazue Mizumura $^{3} \cdot$ Kiyomi Hori $^{1}$ • \\ Noriyuki Ozaki ${ }^{1} \cdot$ Tomoko Koeda $^{2}$
}

Received: 19 June 2017/ Accepted: 2 October 2017/Published online: 12 October 2017

(C) The Physiological Society of Japan and Springer Japan KK 2017

\begin{abstract}
We focused on the analgesic effect of hot packs for mechanical hyperalgesia in physically inactive rats. Male Wistar rats were randomly divided into four groups: control, physical inactivity (PI), PI + sham treatment $(\mathrm{PI}+$ sham), and PI + hot pack treatment $(\mathrm{PI}+$ hot pack) groups. Physical inactivity rats wore casts on both hind limbs in full plantar flexed position for 4 weeks. Hot pack treatment was performed for $20 \mathrm{~min}$ a day, 5 days a week. Although mechanical hyperalgesia and the up-regulation of NGF in the plantar skin and gastrocnemius muscle were observed in the PI and the PI + sham groups, these changes were significantly suppressed in the PI + hot pack group. The present results clearly demonstrated that hot pack treatment was effective in reducing physical inactivity-induced mechanical hyperalgesia and up-regulation of NGF in plantar skin and gastrocnemius muscle.
\end{abstract}

Keywords Physical inactivity - Mechanical hyperalgesia · Nerve growth factor $\cdot$ Thermal therapy $\cdot$ Analgesia

Tomoko Koeda

tomokoed@ngu.ac.jp

1 Department of Functional Anatomy, Graduate School of Medical Science, Kanazawa University, 13-1 Takara-machi, Kanazawa, Ishikawa 920-8640, Japan

2 Faculty of Rehabilitation Sciences, Department of Physical Therapy, Nagoya Gakuin University, 1350 Kamishinano-cho, Seto 480-1298, Japan

3 Department of Physical Therapy, College of Life and Health Sciences, Chubu University, 1200 Matsumoto-cho, Kasugai, Japan

\section{Introduction}

Persons who have health problems treated by casting or bed rest often become physically inactive. However, this may cause undesirable side effects. A recent study showed that long-term bed rest induced disuse atrophy, muscle damage, and pain [1]. In other physically inactive conditions, casting also induced disuse atrophy, joint contracture and cutaneous mechanical and/or cold hyperalgesia in human and animal studies [2-5]. These studies suggest that some physically inactive conditions induce some pain conditions. Muscular atrophy caused by cast immobilization induces the infiltration of macrophages in the skeletal muscle [6]. Macrophages release nerve growth factor (NGF) [7]. NGF is known to be involved in cutaneous tactile allodynia and muscular mechanical hyperalgesia in some disease states [8-13]. An increase in NGF protein in the plantar skin and the up-regulation of NGF positive neurons in the dorsal root ganglions (DRGs) have also been shown to be induced by cast immobilization [14-16]. Furthermore, we have reported that physical inactivity increases the amount of NGF protein in the gastrocnemius muscle [17]. These results suggest that NGF may play a pivotal role in the skin and muscular mechanical hyperalgesia induced by physical inactivity.

Exercise and manual therapy have been reported to be highly effective analgesic therapies in clinical studies [18-21]. However, pain induced by joint movement in physically inactive patients may limit their exercise or the manual therapy they can receive. Thermal therapy is also well known to be useful for treating pain in humans [22-25]. Innocuous heat stimulus increases the tissue temperature, resulting in increased blood flow and tissue metabolism [26]. Increasing blood flow facilitates the supply of nutrients and oxygen to the site of wounds, and 
results in tissue healing $[27,28]$. Tissue healing is believed to accelerate pain reduction. However, the histological and biochemical/molecular changes induced by innocuous heat treatment have not been well studied, and the mechanism of this analgesic action remains largely unknown. In the present experiment, we investigated the effects of thermal treatment with hot packs on mechanical hyperalgesia and the expression of nerve growth factor in the skin and muscle in an animal model of physical inactivity. A preliminary result has been presented in abstract form [17].

\section{Materials and methods}

\section{Animals}

All experiments were conducted with the approval of the animal experiment committees of Nagoya Gakuin University (approval number: 2007-004) and Kanazawa University (approval number: AP-153588), and in accordance with the Ethical Guidelines of the International Association for the Study of Pain.

Male Wistar rats ( $n=38$, aged 8 weeks) weighing $260 \mathrm{~g}$ at the beginning of the experiments were used in this study. These rats were randomly divided into four groups: naive control (CON, $n=10$ ); physical inactivity (PI, $n=10)$; physical inactivity and sham treatment (PI + sham, $n=8)$; and physical inactivity and hot pack treatment $(\mathrm{PI}+$ hot pack, $n=10)$. The rats were housed in plastic cages with an ambient temperature of $23 \pm 1{ }^{\circ} \mathrm{C}$ and maintained on a 12-h light/dark cycle. They had free access to food and water.

\section{Physical inactivity model}

Rats in the PI, PI + sham, and PI + hot pack groups were cast immobilized for 4 weeks. They were anesthetized with isoflurane $(2-4 \%$ in the air, delivered through a mask, Univentor, Zejtun, Malta), and their two hind limbs were encased by plaster casts (ALCARE Co, Ltd, Tokyo) in full plantar flexed position for 4 weeks (Fig. 1a, b). These casts were removed for about $2 \mathrm{~h}$ every day for 5 days per week under anesthesia for hot pack/sham application and withdrawal threshold measurements, after which the casts were put back on (Fig. 1d).

\section{Treatment}

After the temporary cast removal, the rats in the PI + sham and the PI + hot pack group were treated with a hot pack (Hildpack-L, Maruho Corporation, Osaka, Japan). The rats in the PI + hot pack group were anesthetized with isoflurane and laid on a hot pack that had been heated in hot water $\left(50^{\circ} \mathrm{C}\right)$ with their right side down (Fig. 1a, c). The rats in the PI + sham group were treated with a hot pack of the room temperature as a control. These treatments were performed for 20 min 5 times per week.

\section{Skin temperature measurement}

We measured skin temperatures using infrared thermometers (HORIBA, Ltd, Kyoto, Japan) during the casting period. The skin surface temperature was measured shortly after cast removal (within $60 \mathrm{~s}$ ) and then once a week under anesthesia at the heel and the head of fibula in the right hind limb in each group. Apart from this, to get a preliminary rough estimate of the temperature change induced by hot pack treatment, the skin temperature was measured once before and after the hot pack $(n=10)$ or sham treatment $(n=8)$ in the naïve rats.

\section{Mechanical withdrawal threshold measurements}

After cast removal and treatment, we measured the mechanical withdrawal threshold (Fig. 1d). Rats were placed in a modular animal enclosure with a wire mesh bottom $(20 \times 20 \times 13.5 \mathrm{~cm})$, and acclimated for 20-30 min before being subjected to the von Frey test. After acclimation, mechanical stimulus was delivered to the plantar surface of the right hind paw by pressing a 0.5 mm-diameter filament using a Dynamic Plantar Aesthesiometer (Ugo Basile, Comerio, Italy) positioned beneath the bottom of the enclosure. The filament delivered an increasing upward force from 0 to $490 \mathrm{mN}$ over $10 \mathrm{~s}$. The force at which a paw withdrawal response occurred was recorded. Measurements were repeated 5 times at 2-min intervals. The minimum and maximum values were excluded, and the average of the remaining three values was calculated and taken as the withdrawal threshold of the skin.

A Randall-Selitto analgesiometer (Ugo Basile, Comerio, Italy) equipped with a probe of tip diameter $2.6 \mathrm{~mm}$ was used to measure the withdrawal threshold of the right gastrocnemius muscle. Use of a large diameter probe enabled measurement of the withdrawal threshold of the deep tissue [29]. The nociceptive threshold was defined as the force that induced a withdrawal response to an increasing pressure stimulus from 0 to $2450 \mathrm{mN}$. Measurements were repeated 5 times at 1-min intervals, and the average of three values after excluding the minimum and maximum values was taken as the withdrawal threshold of the rat in each experimental session. 
A

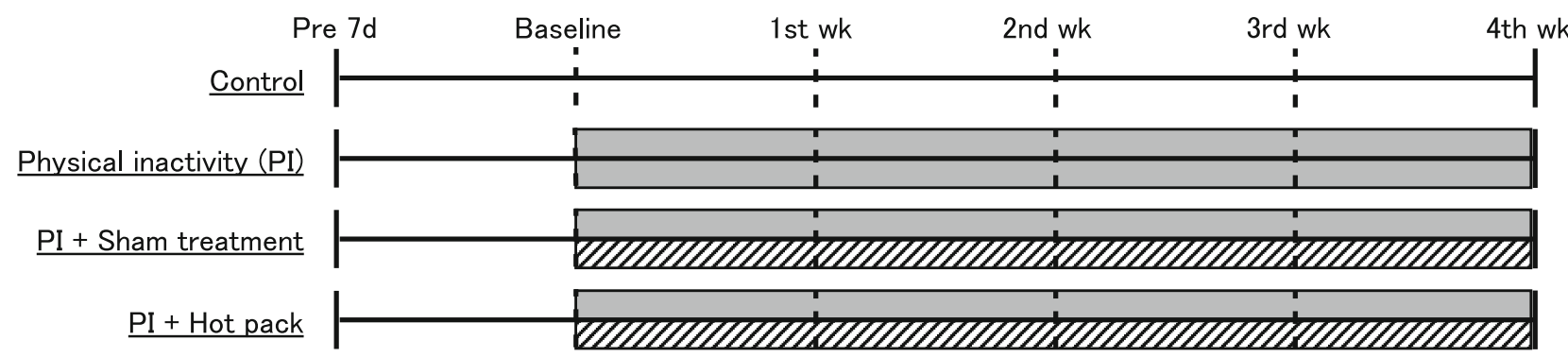

$\square$ : Physical Inactivity period

Sham or hot pack treatment (20 min a day, 5 days a week)

B

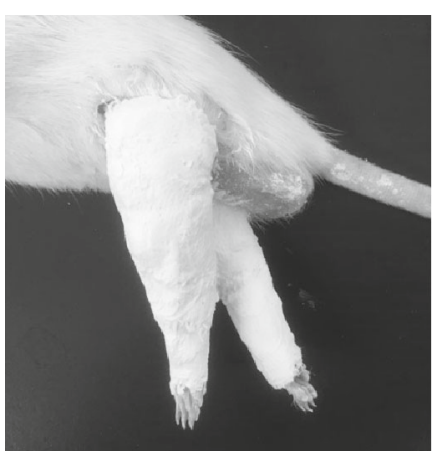

C

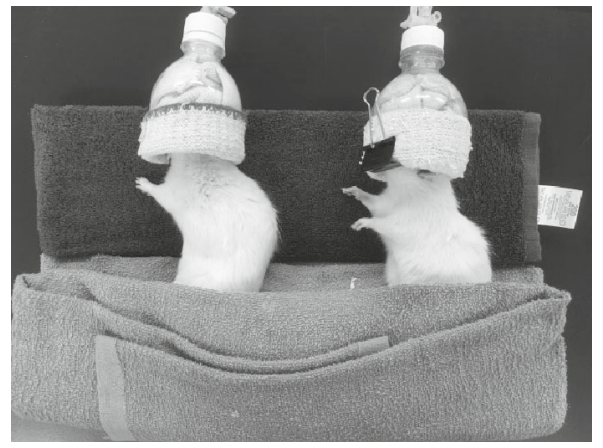

D

\section{Daily protocol}

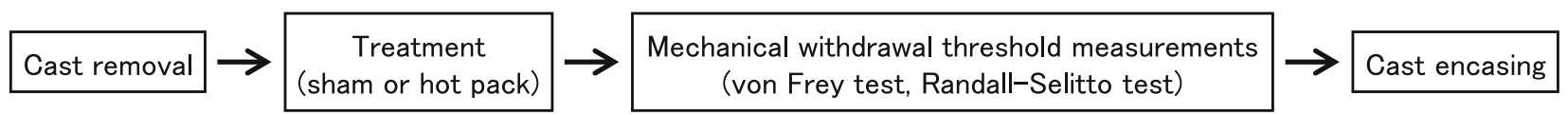

Fig. 1 Hot pack treatment protocols for the experimental groups. a Treatment groups and time schedule of the treatment. Rats were divided into four groups: naïve control $(\mathrm{CON})$, physical inactivity (PI), PI + sham, and PI + hot pack. b Photograph of casted hind limbs. Both hind limbs of rats in the PI, PI + sham, and PI + hot pack group were immobilized in full plantar flexed position using plaster casts for 4 weeks. c Photograph showing how hot pack was applied to rats. PI + sham and PI + hot pack groups were

\section{Enzyme-linked immunosorbent assay for NGF}

At the end of experiment, the plantar skin and gastrocnemius muscle were excised under anesthesia (pentobarbital sodium, i.p. $50 \mathrm{mg} / \mathrm{kg}$ ) and quickly frozen in liquid nitrogen. The frozen plantar skin and gastrocnemius muscle were crushed into small pieces using a pestle and mortar. The pieces of the plantar skin and gastrocnemius muscle were homogenized 1:10 (w/v) in lysis buffer $(25 \mathrm{mM}$ Tris$\mathrm{HCl}, 150 \mathrm{mM} \mathrm{NaCl}, 1 \% \mathrm{NP}-40,0.5 \%$ sodium deoxycholate, and protease inhibitor in distilled water). Homogenates were centrifuged for $15 \mathrm{~min}$ at $15,000 \mathrm{rpm}$ at $4{ }^{\circ} \mathrm{C}$. Supernatants were preserved at $-80^{\circ} \mathrm{C}$ until assay. NGF anesthetized with isoflurane and placed in a left lateral position on the hot pack and covered for 20 min a day, 5 days per week. d Daily protocol. Firstly, casts were removed from rats in the PI + sham and the PI + hot pack groups. Secondly, they received the sham or hot pack treatment. Next, their mechanical withdrawal threshold was examined. Finally, casts were put back on both hind limbs. In the PI group, the withdrawal threshold was measured after cast removal

protein levels were measured using a rat beta-NGF DuoSet ELISA kit (DY556, R\&D Systems, Inc., Minneapolis, MN, USA) and total protein levels using a BCA protein assay kit (Thermo Fisher Scientific Inc., Waltham, MA, USA), according to the manufacturers' protocols.

\section{Statistical analysis}

All data are presented as the mean \pm SEM. Statistical analyses were performed using SigmaPlot 13 (Systat Software Inc., San Jose, CA, USA). Skin temperatures and withdrawal thresholds were analyzed by two-way repeated measures analysis of variance (ANOVA) followed by 
Holm-Sidak multiple comparison tests. NGF protein was analyzed by one-way ANOVA followed by Holm-Sidak multiple comparison test. Results were considered statistically significant when $p<0.05$.

\section{Results}

\section{Change of skin temperature after hot pack therapy}

The skin temperatures at the heel and the head of the fibula in each group are chronologically shown in Table 1 . There was no main effect of interaction and no significant difference in the temperatures among the four groups or five measurements at the heel ( $p>0.05$, respectively) although there was a significant difference among four groups at the head of the fibula $(p<0.05)$. However, there was no main effect of interaction $(p>0.05)$.

In a preliminary experiment using some naïve rats, the skin temperature was separately measured before and after the hot pack and sham treatments. After treatment, the temperature of the skin at the right heel remained at almost the same level $\left(29.9 \pm 1.2^{\circ} \mathrm{C}\right)$ with the sham treatment of naïve rats $(p>0.05)$ and increased to $35.1 \pm 0.6{ }^{\circ} \mathrm{C}$ with the hot pack treatment of naïve rats ( $p<0.05$, Fig. 2$)$. The temperature of the skin at the head of the right fibula was $31.3 \pm 1.3{ }^{\circ} \mathrm{C}$ with the sham treatment of naïve rats and $35.7 \pm 0.5^{\circ} \mathrm{C}$ with the hot pack treatment of naïve rats. The skin temperature after treatment with the hot pack treatment of naïve rats was significantly higher in both the heel and the head of the fibula compared with the sham treatment of naïve rats $(p<0.05)$.

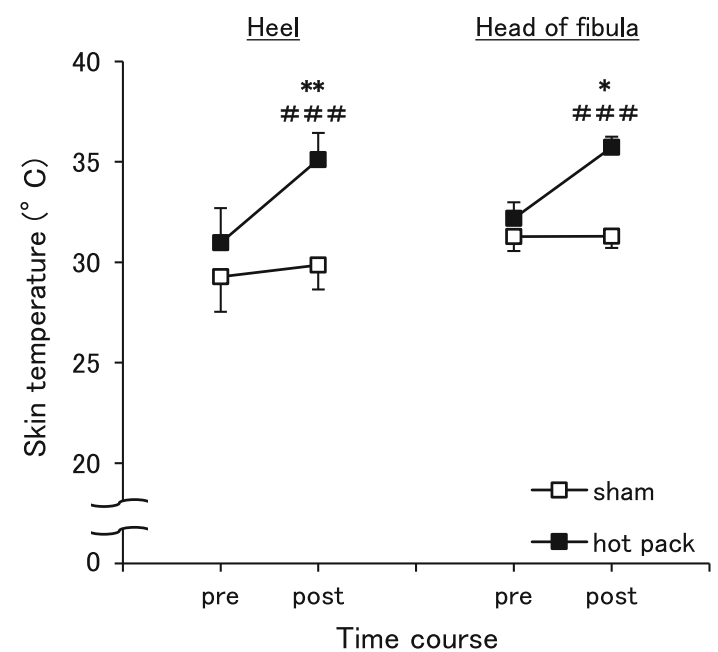

Fig. 2 Changes in skin temperature after treatment. The skin temperatures of the right heel (left graph) and the head of fibula (right graph) were significantly increased after the hot pack treatment compared to the sham treatment. $* p<0.05$ and $* * p<0.01$ vs the sham treatment. ${ }^{\# \#} p<0.001$ vs pre within the hot pack treatment

\section{Hot pack therapy suppressed the development of mechanical hyperalgesia induced by physical inactivity}

There were significant differences among the four groups or five measurements for the mechanical withdrawal thresholds of the plantar skin ( $p<0.001$, respectively). There was also a significant interaction between groups and measurements $(p<0.01)$. The mechanical withdrawal thresholds of the plantar skin in the PI group gradually decreased from 1 week after casting compared with baseline (BL), while that in the $\mathrm{CON}$ group remained unchanged $(p<0.05-0.001$, Fig. 3a). The withdrawal threshold in the PI + sham group decreased similarly to that in the PI group. The withdrawal thresholds of both groups were significantly lower than that of the CON group
Table 1 Skin temperatures before treatment at the heel and head of fibula in each group

\begin{tabular}{|c|c|c|c|c|c|c|}
\hline & \multirow[t]{2}{*}{ Group } & \multicolumn{5}{|c|}{ Skin temperatures } \\
\hline & & Baseline & 1st week & 2nd week & 3rd week & 4th week \\
\hline \multirow[t]{4}{*}{ Heel } & $\mathrm{CON}$ & $30.2 \pm 1.3$ & $28.3 \pm 0.9$ & $25.9 \pm 0.6$ & $27.9 \pm 1.3$ & $26.6 \pm 1.1$ \\
\hline & PI & $29.5 \pm 1.4$ & $28.2 \pm 1.1$ & $27.3 \pm 0.6$ & $27.4 \pm 1.0$ & $25.5 \pm 0.7$ \\
\hline & PI + sham & $29.2 \pm 1.8$ & $28.1 \pm 1.4$ & $28.7 \pm 1.2$ & $28.6 \pm 1.9$ & $25.3 \pm 0.3$ \\
\hline & PI + hot pack & $31.1 \pm 0.9$ & $28.2 \pm 1.1$ & $25.7 \pm 1.1$ & $29.8 \pm 1.5$ & $27.4 \pm 0.9$ \\
\hline \multirow[t]{4}{*}{ Head of fibula } & $\mathrm{CON}$ & $33.8 \pm 0.2$ & $33.6 \pm 0.2$ & $33.0 \pm 1.0$ & $33.9 \pm 0.2$ & $33.7 \pm 0.4$ \\
\hline & PI & $33.5 \pm 0.6$ & $32.2 \pm 0.6$ & $31.5 \pm 0.8$ & $32.2 \pm 0.3$ & $31.7 \pm 0.5$ \\
\hline & PI + sham & $31.9 \pm 0.8$ & $32.1 \pm 0.6$ & $31.5 \pm 0.6$ & $32.6 \pm 0.6$ & $31.7 \pm 0.4$ \\
\hline & PI + hot pack & $33.8 \pm 0.3$ & $33.4 \pm 0.6$ & $32.6 \pm 1.1$ & $33.1 \pm 0.7$ & $32.4 \pm 0.5$ \\
\hline
\end{tabular}

We observed the chronological change of skin temperatures due to physical inactivity or treatment. Skin temperatures were taken soon after cast removal. Skin temperatures are expressed as mean \pm SEM. There were no significant differences between any groups during the physical inactivity period 
Fig. 3 Hot pack treatment attenuated the physical inactivity-induced cutaneous and muscular mechanical hyperalgesia. a von Frey test and b Randall-Selitto test were performed to measure the cutaneous and muscular mechanical withdrawal thresholds from baseline (BL) to 4th week. The decrease in the cutaneous and muscular mechanical withdrawal thresholds in the PI and $\mathrm{PI}+$ sham groups was not seen to the same extent in the PI + hot pack group. $* p<0.05, * * p<0.01$, and $* * * p<0.001$ vs CON group. ${ }^{\#} p<0.05,{ }^{\#} p<0.01$, and $\# \#$ $p<0.001$ vs PI group. ${ }^{\dagger}$ $p<0.05,{ }^{\dagger \dagger} p<0.01$, and ${ }^{\dagger \dagger}$ $p<0.001$ vs $\mathrm{PI}+$ sham group

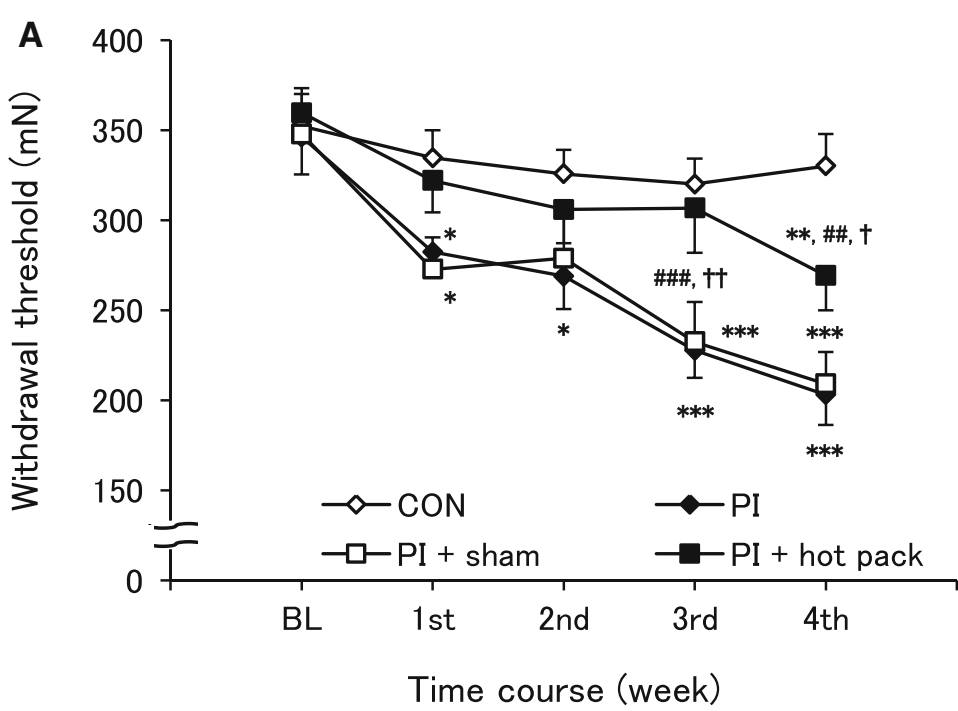

B

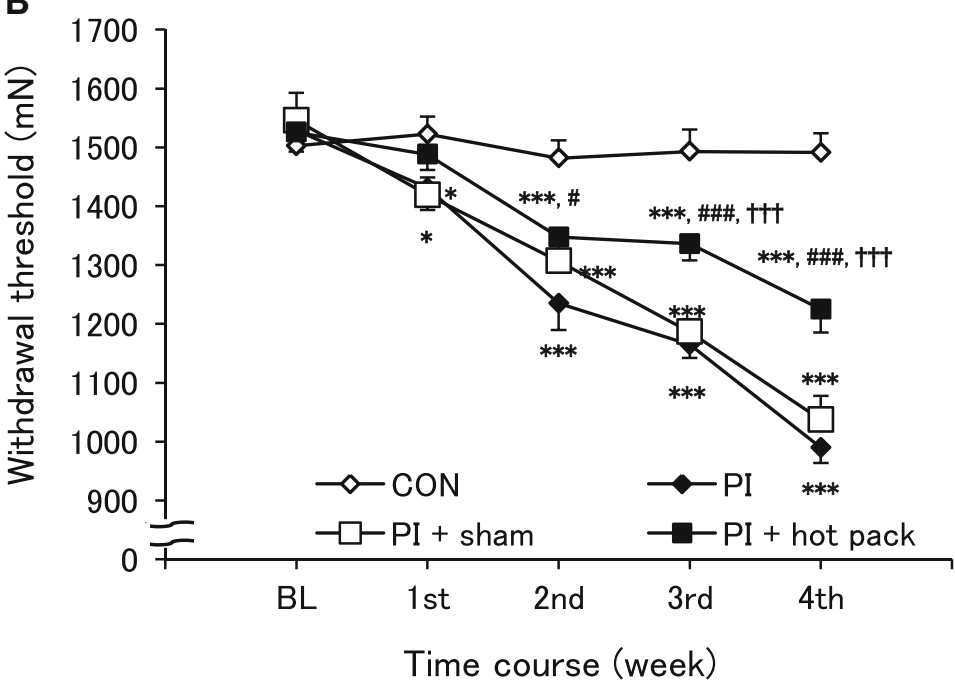

at all time points except the baseline. In contrast, the cutaneous mechanical withdrawal threshold of the PI + hot pack group showed almost no decrease except in the 1 st week, and there were significant differences from those in the PI and PI + sham groups. It should be noted that the threshold of the PI + hot pack group was not significantly different from that of the CON group except in the 4th week. In the 4th week, the threshold of the $\mathrm{PI}+$ hot pack group was lower than that of the CON group, yet it was still significantly higher than those in the PI and the PI + sham groups.

In the gastrocnemius muscle, there were also significant differences in the mechanical withdrawal threshold among the four groups or five measurements or interaction ( $p<0.001$, respectively). The mechanical withdrawal threshold in the PI group significantly decreased in a stepwise fashion from the 1 st week to the 4th week $(p<0.001$, Fig. 3b). That in the $\mathrm{PI}+$ sham group decreased similarly. The muscular mechanical withdrawal threshold of the PI + hot pack group also decreased but, unlike the skin withdrawal threshold, it was significantly higher than those of the PI (except at the 1st week) and PI + sham (at the 3rd and 4th weeks) groups, and significantly lower than that of the CON group except in the 1st week.

\section{Hot pack therapy suppressed the physical inactivity- induced up-regulation of NGF}

At the end of the physical inactivity period, NGF protein in the plantar skin and gastrocnemius muscle were measured, and there were significant differences in NGF protein in the plantar skin $(p<0.01)$ and gastrocnemius muscle $(p<0.001)$ among the 4 groups. It was significantly increased in the PI and the PI + sham groups compared with the CON group ( $p<0.05-0.001$, Fig. 4a, b). No 

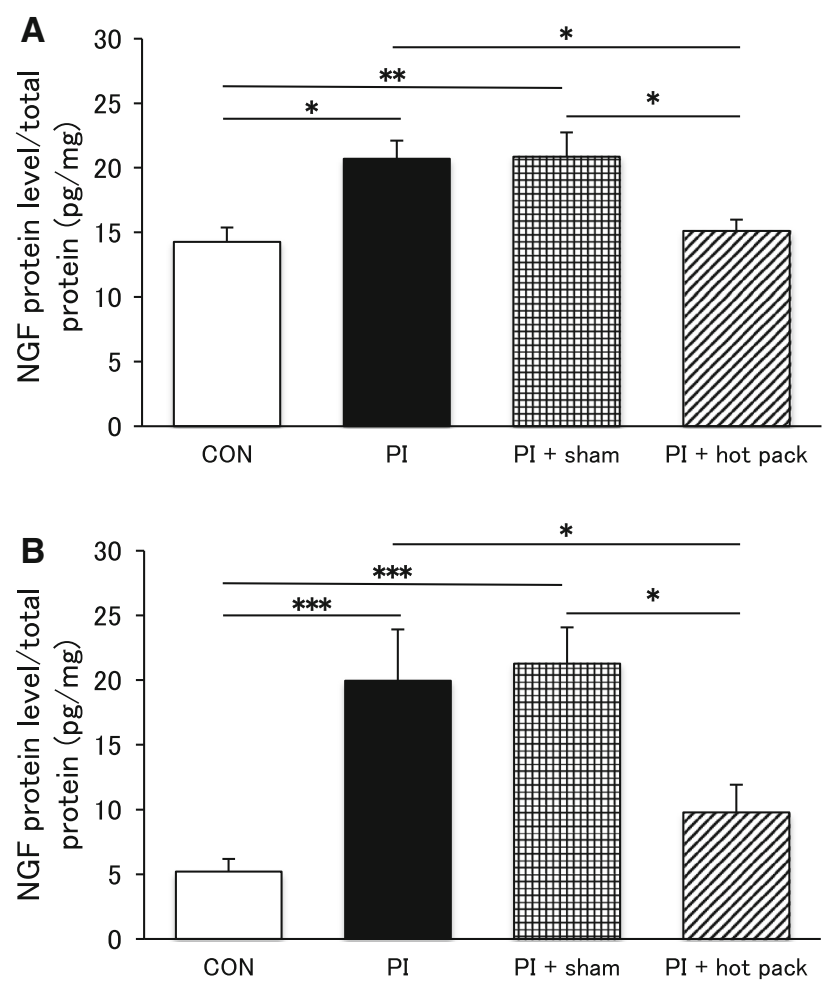

Fig. 4 Hot pack inhibited the up-regulation of NGF in the plantar skin and gastrocnemius muscle. NGF protein levels of the a plantar skin and $\mathbf{b}$ gastrocnemius muscle in the PI + hot pack group were significantly lower than the PI and the PI + sham group. No significant difference was observed between the PI and the PI + sham group. This elevated NGF level was suppressed by hot pack treatment. $* p<0.05, * * p<0.01$ and $* * * p<0.001$ vs each other

significant difference was observed between the PI and the PI + sham groups. In contrast, NGF in the PI + hot pack group was significantly lower than that in the PI and PI + sham groups $(p<0.05)$, and it was not different from that in the CON group, indicating that the increase in NGF as a result immobility was impeded by the hot pack.

\section{Discussion}

Rats with both hind limbs immobilized by cast were used as a physical inactivity model in this study. It has been previously reported that this model shows muscle atrophy and joint contracture that are similar to changes induced by bed rest [30]. Thus, our model mimics a bed rest condition. Physically inactive rats showed decreased cutaneous and muscular mechanical withdrawal thresholds, which are conditions compatible with human cutaneous and muscle hyperalgesia. We measured withdrawal threshold in the gastrocnemius muscle using a 2.6-mm-diameter probe. A previous study showed that a large probe can measure deep tissue withdrawal thresholds excluding the effect of skin [29]. In this study, our results show muscle withdrawal threshold but not skin withdrawal threshold. They also showed up-regulation of NGF in the plantar skin and gastrocnemius muscle. It has been reported that NGF is responsible for mechanical hyperalgesia $[8,9,11]$. Murase et al. reported that the extensor digitorum longus (EDL) muscle of rats with delayed onset muscle soreness showed mechanical hyperalgesia, that NGF was up-regulated in a time course compatible with mechanical hyperalgesia, and that this mechanical hyperalgesia was reversed by the intramuscular injection of anti-NGF antibody [31]. We also previously reported that muscular mechanical hyperalgesia induced by physical inactivity was suppressed by anti-NGF antibody [17]. In addition to that result, our present finding that physical inactivity induced an increase in NGF protein and muscular mechanical hyperalgesia in the gastrocnemius muscle indicates that NGF contributes to physical inactivity-induced mechanical muscular hyperalgesia.

Furthermore, we found that hot pack treatment inhibited, though not completely, physical inactivity-induced cutaneous and muscular mechanical hyperalgesia, and that the hot pack also reduced the up-regulation of NGF protein levels. In contrast, NGF in the PI + sham group was not different from that in the PI group. We examined whether hot pack stimulation is noxious or innocuous heat stimulation in a preliminary study. The maximum temperature of the hot pack during treatment was $40.2 \pm 0.4{ }^{\circ} \mathrm{C}$. Therefore, this stimulation is presumed to be innocuous heat based on results from our preliminary study. These results suggest that the NGF suppressing effect was produced by the innocuous heat stimulation, not by the tactile or pressure stimulation from the hot pack material. It is known that heat stimuli induce an increase in blood flow to dilate vessels and increase their permeability [32]. Song et al. found that the blood flow in the skin was increased 15-fold compared with the control value while that in the muscle was also increased ninefold when rodent legs were warmed in a $45{ }^{\circ} \mathrm{C}$ water bath [33]. Noguchi et al. have also reported that moxibustion-like thermal stimulation for rats induced a $23 \%$ increase in the blood flow in the gastrocnemius muscle, which was maintained for 14 min after treatment [34]. In a preliminary study, we checked the change in muscle temperature after hot pack application in a rat by inserting a thermoprobe into the muscle. The muscle temperature of the gastrocnemius muscle was increased from 33.4 to $36.7^{\circ} \mathrm{C}$ after treatment, which was smaller than the change in the skin. This might be the reason that the change in the withdrawal threshold with a hot pack was smaller in the muscle. Hot pack treatment is thought to regulate the blood flow and metabolism in the skin and the muscle, and consequently the elevated blood flow may play a role in the reduction of NGF.

The present study showed that the cutaneous mechanical withdrawal threshold in the PI + hot pack group was 
significantly lower than that in the CON group even though NGF protein in the PI + hot pack group was at the same level as that in the CON group at the end of experiment. Some mechanisms by which NGF induces pain have been reported [35]. In the acute phase, NGF sensitizes the transient receptor potential vanilloid receptor 1 (TRPV1) through the TrkA receptor which has the highest binding affinity for NGF, and this sensitization is related to hyperalgesia [36, 37]. NGF also activates mast cells, and activated mast cells release some pain mediators [38]. These changes also induce pain. Other mechanisms are the retrograde transport of NGF from peripheral nerve endings to DRGs [39], leading to transcriptional changes that result in the up-regulation of nociceptive proteins such as TRPV1, brain-derived neurotrophic factor (BDNF), and acid-sensing ion channel 3 (ASIC3) [40-42]. The average propagation speed of NGF within the axon is reported to be $1.3 \mu \mathrm{m} / \mathrm{s}$ [43], indicating that the up-regulation of some nociceptive proteins induced by NGF does not occur quickly. The difference between behavioral and biological results hints at the possibility that hot pack treatment induces temporary NGF deletion and suppresses the hyperalgesia induced by the activation of mast cells and the sensitization of TRPV1, but does not suppress the hyperalgesia induced by up-regulations of nociceptors. We also performed the behavioral pharmacology experiments investigating transient receptor potential vanilloid receptor 4 (TRPV4) and protease activated receptor 2 (PAR2), because it has recently been shown that these substances may involve mechanical hyperalgesia [44, 45]. Dose-dependent suppression of the mechanical hyperalgesia was observed after administration of TRPV4 or PAR2 antagonists, respectively, at the end of the physical inactivity period. Moreover, no significant differences were detected after injection of TRPV1 antagonist or ASIC3 antagonist [46]. PAR2 and TRPV4 may relate in physical inactivityinduced mechanical hyperalgesia.

A recent report showed that heat stimulation facilitated inflammatory reactions after muscle injury, resulting in the acceleration of muscle recovery and reduction in inflammatory reactions [47]. Some reports have investigated the effect of thermal therapy using molecular biological methods. They have shown that repeated thermal therapy up-regulated heat shock protein (HSP) [48-50]. HSPs facilitate the folding and unfolding of proteins, protein translocation, and anti-apoptotic mechanisms [51]. Anti-inflammatory roles of HSP have also been described [52]. HSP induction down-regulated the expressions of IL- 8 and TNF- $\alpha$ mRNA and the protein levels of these cytokines [53]. In addition, heat shock factor 1 , which is a major transcription activator of the heat shock gene, suppresses the transcription of prointerleukin 1- $\beta$ [54]. It has been noted that TNF- $\alpha$ and IL-
$1 \beta$ induced an increase in NGF in keratinocytes $[11,55]$. It is likely that thermal therapy has a suppressive effect on inflammation, and represses the up-regulation of NGF. For the moment, however, we have no data on the expression of HSP, TNF- $\alpha$, and IL-1 $\beta$. Further studies will be necessary to establish the mechanism of thermal therapy-induced analgesia.

Thermal therapy using hot packs is commonly used for treatment of pain. However, the analgesic effect is uncertain. Our study clearly demonstrates that hot pack treatment improves physical inactivity-induced mechanical hyperalgesia and up-regulation of NGF. NGF is known to be associated with some pain conditions, such as delayed onset muscle soreness, bone injury, diabetes, inflammation, nerve injury, osteoarthritis and low back pain [9-11, 31, 56-58]. Tanezumab is a humanized monoclonal antibody that specifically inhibits nerve growth factor and has recently become a focus as a new treatment for chronic pain [58-60]. However, the safety this drug has not yet been established because the lack of NGF induces some side effects, such as headache, upper respiratory tract infection, and paresthesia [59]. These results suggest that hot pack treatment may be an effective analgesic treatment for musculoskeletal pain, with the exception of some side effects.

Finally, we clearly demonstrated that hot pack treatment was effective in reducing physical inactivity-induced mechanical hyperalgesia and up-regulation of NGF in plantar skin and gastrocnemius muscle. The mechanisms of this hyperalgesia, NGF up-regulation and their suppression by hot pack treatment remain open for future study.

\section{Compliance with ethical standards}

Funding This work was supported by JSPS KAKENHI Grant Numbers JP23500624 and JP26350638.

Conflict of interest The authors have no conflicts of interest to declare.

Ethical approval All experiments were conducted with the approval of the animal experiment committees of Nagoya Gakuin University (Approval Number: 2007-004) and Kanazawa University (Approval Number: AP-153588), and in accordance with the Ethical Guidelines of the International Association for the Study of Pain. This article does not contain any studies with human participants performed by any of the authors.

\section{References}

1. Miokovic T, Armbrecht G, Gast U, Rawer R, Roth HJ, Runge M, Felsenberg D, Belavy DL (2014) Muscle atrophy, pain, and damage in bed rest reduced by resistive (vibration) exercise. Med Sci Sports Exerc 46:1506-1516 
2. Terkelsen AJ, Bach FW, Jensen TS (2008) Experimental forearm immobilization in humans induces cold and mechanical hyperalgesia. Anesthesiology 109:297-307

3. Hamaue Y, Nakano J, Sekino Y, Chuganji S, Sakamoto J, Yoshimura T, Origuchi T, Okita M (2013) Immobilization-induced hypersensitivity associated with spinal cord sensitization during cast immobilization and after cast removal in rats. J Physiol Sci 63:401-408

4. Psatha M, Wu Z, Gammie FM, Ratkevicius A, Wackerhage H, Lee JH, Redpath TW, Gilbert FJ, Ashcroft GP, Meakin JR (2012) A longitudinal MRI study of muscle atrophy during lower leg immobilization following ankle fracture. J Magn Reson Imaging 35:686-695

5. Morimoto A, Winaga H, Sakurai H, Ohmichi M, Yoshimoto T, Ohmichi Y, Matsui T, Ushida T, Okada T, Sato J (2013) Treadmill running and static stretching improve long-lasting hyperalgesia, joint limitation, and muscle atrophy induced by cast immobilization in rats. Neurosci Lett 534:295-300

6. Zhu S, Nagashima M, Khan MA, Yasuhara S, Kaneki M, Martyn JA (2013) Lack of caspase-3 attenuates immobilization-induced muscle atrophy and loss of tension generation along with mitigation of apoptosis and inflammation. Muscle Nerve 47:711-721

7. Takano S, Uchida K, Miyagi M, Inoue G, Fujimaki H, Aikawa J, Iwase D, Minatani A, Iwabuchi K, Takaso M (2016) Nerve growth factor regulation by TNF-alpha and IL-1beta in synovial macrophages and fibroblasts in osteoarthritic mice. J Immunol Res 2016:5706359

8. Hayashi K, Ozaki N, Kawakita K, Itoh K, Mizumura K, Furukawa K, Yasui M, Hori K, Yi SQ, Yamaguchi T, Sugiura Y (2011) Involvement of NGF in the rat model of persistent muscle pain associated with taut band. J Pain 12:1059-1068

9. Cheng HT, Dauch JR, Hayes JM, Hong Y, Feldman EL (2009) Nerve growth factor mediates mechanical allodynia in a mouse model of type 2 diabetes. J Neuropathol Exp Neurol 68:1229-1243

10. Ro LS, Chen ST, Tang LM, Jacobs JM (1999) Effect of NGF and anti-NGF on neuropathic pain in rats following chronic constriction injury of the sciatic nerve. Pain 79:265-274

11. Safieh-Garabedian B, Poole S, Allchorne A, Winter J, Woolf CJ (1995) Contribution of interleukin-1 beta to the inflammationinduced increase in nerve growth factor levels and inflammatory hyperalgesia. Br J Pharmacol 115:1265-1275

12. Hayashi K, Shiozawa S, Ozaki N, Mizumura K, Graven-Nielsen $\mathrm{T}$ (2013) Repeated intramuscular injections of nerve growth factor induced progressive muscle hyperalgesia, facilitated temporal summation, and expanded pain areas. Pain 154:2344-2352

13. Deising S, Weinkauf B, Blunk J, Obreja O, Schmelz M, Rukwied R (2012) NGF-evoked sensitization of muscle fascia nociceptors in humans. Pain 153:1673-1679

14. Sekino Y, Nakano J, Hamaue Y, Chuganji S, Sakamoto J, Yoshimura T, Origuchi T, Okita M (2014) Sensory hyperinnervation and increase in NGF, TRPV1 and P2X3 expression in the epidermis following cast immobilization in rats. Eur $\mathrm{J}$ Pain 18:639-648

15. Nishigami T, Osako Y, Ikeuchi M, Yuri K, Ushida T (2013) Development of heat hyperalgesia and changes of TRPV1 and NGF expression in rat dorsal root ganglion following joint immobilization. Physiol Res 62:215-219

16. Guo TZ, Wei T, Li WW, Li XQ, Clark JD, Kingery WS (2014) Immobilization contributes to exaggerated neuropeptide signaling, inflammatory changes, and nociceptive sensitization after fracture in rats. J Pain 15:1033-1045

17. Hiraga S, Koeda $T$, Hori $K$, Nakata $H$, Nakagawa $T$, Iseki $S$, Ozaki N (2016) NGF increased in skeletal muscle has a role in muscular mechanical hyperalgesia in inactivity model. 16th World Congress on Pain Yokohama, JPN
18. Chou R, Qaseem A, Snow V, Casey D, Cross JT Jr, Shekelle P, Owens DK (2007) Diagnosis and treatment of low back pain: a joint clinical practice guideline from the American College of Physicians and the American Pain Society. Ann Intern Med 147:478-491

19. Koes BW, van Tulder M, Lin C-WC, Macedo LG, McAuley J, Maher C (2010) An updated overview of clinical guidelines for the management of non-specific low back pain in primary care. Eur Spine J 19:2075-2094

20. Sluka KA, Milosavljevic S (2016) Manual therapy. In: Sluka KA (ed) Mechanisms and management of pain for the physical therapist. Wolters Kluwer, Philadelphia

21. Bement MH, Sluka KA (2016) Exercise-induced hypoalogesia: an evidence-based review. In: Sluka KA (ed) Mechanisms and management of pain for the physical therapist. Wolters Kluwer, Philadelphia

22. Baxter GD, Basford JR (2016) Overview of other electrophysical agents including thermal modalities. In: Sluka KA (ed) Mechanisms and management of pain for the physical therapist. Wolters Kluwer, Philadelphia

23. Masuda A, Hattanmaru M, Tei C (2006) Repeated thermal therapy improves outcomes in patients with chronic pain. Int Congr Ser 1287:298-303

24. Masuda A, Koga Y, Hattanmaru M, Minagoe S, Tei C (2005) The effects of repeated thermal therapy for patients with chronic pain. Psychother Psychosom 74:288-294

25. Mayer JM, Mooney V, Matheson LN, Erasala GN, Verna JL, Udermann BE, Leggett S (2006) Continuous low-level heat wrap therapy for the prevention and early phase treatment of delayedonset muscle soreness of the low back: a randomized controlled trial. Arch Phys Med Rehabil 87:1310-1317

26. Nadler SF, Weingand K, Kruse RJ (2004) The physiologic basis and clinical applications of cryotherapy and thermotherapy for the pain practitioner. Pain Physician 7:395-399

27. Hunt TK, Zederfeldt B, Goldstick TK (1969) Oxygen and healing. Am J Surg 118:521-525

28. Tandara AA, Mustoe TA (2004) Oxygen in wound healingmore than a nutrient. World J Surg 28:294-300

29. Nasu T, Taguchi T, Mizumura K (2010) Persistent deep mechanical hyperalgesia induced by repeated cold stress in rats. Eur J Pain 14:236-244

30. Okita M, Yoshimura T, Nakano J, Motomura M, Eguchi K (2004) Effects of reduced joint mobility on sarcomere length, collagen fibril arrangement in the endomysium, and hyaluronan in rat soleus muscle. J Muscle Res Cell Motil 25:159-166

31. Murase S, Terazawa E, Queme F, Ota H, Matsuda T, Hirate K, Kozaki Y, Katanosaka K, Taguchi T, Urai H, Mizumura K (2010) Bradykinin and nerve growth factor play pivotal roles in muscular mechanical hyperalgesia after exercise (delayed-onset muscle soreness). J Neurosci 30:3752-3761

32. Song CW (1984) Effect of local hyperthermia on blood flow and microenvironment: a review. Cancer Res 44:4721s-4730s

33. Song CW, Kang MS, Rhee JG, Levitt SH (1980) Effect of hyperthermia on vascular function in normal and neoplastic tissues. Ann N Y Acad Sci 335:35-47

34. Noguchi E, Ohsawa H, Takagi K (2009) Neural mechanism of localized changes in skeletal muscle blood flow caused by moxibustion-like thermal stimulation of anesthetized rats. J Physiol Sci 59:421-427

35. Hefti FF, Rosenthal A, Walicke PA, Wyatt S, Vergara G, Shelton DL, Davies AM (2006) Novel class of pain drugs based on antagonism of NGF. Trends Pharmacol Sci 27:85-91

36. Shu X, Mendell LM (1999) Nerve growth factor acutely sensitizes the response of adult rat sensory neurons to capsaicin. Neurosci Lett 274:159-162 
37. Zhu W, Oxford GS (2007) Phosphoinositide-3-kinase and mitogen activated protein kinase signaling pathways mediate acute NGF sensitization of TRPV1. Mol Cell Neurosci 34:689-700

38. Kawamoto K, Aoki J, Tanaka A, Itakura A, Hosono H, Arai H, Kiso Y, Matsuda H (2002) Nerve growth factor activates mast cells through the collaborative interaction with lysophosphatidylserine expressed on the membrane surface of activated platelets. J Immunol 168:6412-6419

39. Delcroix JD, Valletta JS, Wu C, Hunt SJ, Kowal AS, Mobley WC (2003) NGF signaling in sensory neurons: evidence that early endosomes carry NGF retrograde signals. Neuron 39:69-84

40. Mamet J, Baron A, Lazdunski M, Voilley N (2002) Proinflammatory mediators, stimulators of sensory neuron excitability via the expression of acid-sensing ion channels. J Neurosci 22:10662-10670

41. Winston J, Toma H, Shenoy M, Pasricha PJ (2001) Nerve growth factor regulates VR-1 mRNA levels in cultures of adult dorsal root ganglion neurons. Pain 89:181-186

42. Apfel SC, Wright DE, Wiideman AM, Dormia C, Snider WD, Kessler JA (1996) Nerve growth factor regulates the expression of brain-derived neurotrophic factor mRNA in the peripheral nervous system. Mol Cell Neurosci 7:134-142

43. Cui B, Wu C, Chen L, Ramirez A, Bearer EL, Li WP, Mobley WC, Chu S (2007) One at a time, live tracking of NGF axonal transport using quantum dots. Proc Natl Acad Sci USA 104:13666-13671

44. Chen Y, Yang C, Wang ZJ (2011) Proteinase-activated receptor 2 sensitizes transient receptor potential vanilloid 1, transient receptor potential vanilloid 4 , and transient receptor potential ankyrin 1 in paclitaxel-induced neuropathic pain. Neuroscience 193:440-451

45. Ota H, Katanosaka K, Murase S, Kashio M, Tominaga M, Mizumura K (2013) TRPV1 and TRPV4 play pivotal roles in delayed onset muscle soreness. PLoS One 8:e65751

46. Nakagawa T, Koeda T, Hiraga S, Hori K, Ozaki N (2016) TRPV4 with NGF and PAR2 contributes to cutaneous tactile allodynia and muscular mechanical hyperalgesia in a new rat model of physical inactivity. 16th World Congress on Pain Yokohama, JPN

47. Takeuchi K, Hatade T, Wakamiya S, Fujita N, Arakawa T, Miki A (2014) Heat stress promotes skeletal muscle regeneration after crush injury in rats. Acta Histochem 116:327-334

48. Miyauchi T, Miyata M, Ikeda Y, Akasaki Y, Hamada N, Shirasawa T, Furusho Y, Tei C (2012) Waon therapy upregulates Hsp90 and leads to angiogenesis through the Akt-endothelial nitric oxide synthase pathway in mouse hindlimb ischemia. Circ J 76:1712-1721
49. Morimoto Y, Kondo Y, Kataoka H, Honda Y, Kozu R, Sakamoto J, Nakano J, Origuchi T, Yoshimura T, Okita M (2015) Heat treatment inhibits skeletal muscle atrophy of glucocorticoid-induced myopathy in rats. Physiol Res 64:897-905

50. Gatto H, Viac J, Charveron M, Schmitt D (1992) Effects of thermal shocks on interleukin-1 levels and heat shock protein 72 (HSP72) expression in normal human keratinocytes. Arch Dermatol Res 284:414-417

51. Jindal S (1996) Heat shock proteins: applications in health and disease. Trends Biotechnol 14:17-20

52. Chen Y, Voegeli TS, Liu PP, Noble EG, Currie RW (2007) Heat shock paradox and a new role of heat shock proteins and their receptors as anti-inflammation targets. Inflamm Allergy Drug Targets 6:91-100

53. Yoo CG, Lee S, Lee CT, Kim YW, Han SK, Shim YS (2000) Anti-inflammatory effect of heat shock protein induction is related to stabilization of I kappa B alpha through preventing I kappa B kinase activation in respiratory epithelial cells. J Immunol 164:5416-5423

54. Cahill CM, Waterman WR, Xie Y, Auron PE, Calderwood SK (1996) Transcriptional repression of the prointerleukin 1beta gene by heat shock factor 1. J Biol Chem 271:24874-24879

55. Li L, Xian CJ, Zhong JH, Zhou XF (2003) Lumbar 5 ventral root transection-induced upregulation of nerve growth factor in sensory neurons and their target tissues: a mechanism in neuropathic pain. Mol Cell Neurosci 23:232-250

56. Yasui M, Shiraishi Y, Ozaki N, Hayashi K, Hori K, Ichiyanagi M, Sugiura Y (2012) Nerve growth factor and associated nerve sprouting contribute to local mechanical hyperalgesia in a rat model of bone injury. Eur J Pain 16:953-965

57. Xu L, Nwosu LN, Burston JJ, Millns PJ, Sagar DR, Mapp PI, Meesawatsom P, Li L, Bennett AJ, Walsh DA, Chapman V (2016) The anti-NGF antibody muMab 911 both prevents and reverses pain behaviour and subchondral osteoclast numbers in a rat model of osteoarthritis pain. Osteoarthr Cartil 24:1587-1595

58. Leite VF, Buehler AM, El Abd O, Benyamin RM, Pimentel DC, Chen J, Hsing WT, Mazloomdoost D, Amadera JE (2014) Antinerve growth factor in the treatment of low back pain and radiculopathy: a systematic review and a meta-analysis. Pain Physician 17:E45-E60

59. Lane NE, Schnitzer TJ, Birbara CA, Mokhtarani M, Shelton DL, Smith MD, Brown MT (2010) Tanezumab for the treatment of pain from osteoarthritis of the knee. $\mathrm{N}$ Engl $\mathrm{J}$ Med 363:1521-1531

60. Kivitz AJ, Gimbel JS, Bramson C, Nemeth MA, Keller DS, Brown MT, West CR, Verburg KM (2013) Efficacy and safety of tanezumab versus naproxen in the treatment of chronic low back pain. Pain 154:1009-1021 